Original Article

\title{
KNOWLEDGE, ATTITUDES AND PERCEIVED PRACTICE TOWARDS ASTHMA AMONG PHARMACY STUDENTS IN SOUTHERN NIGERIA
}

\author{
KOSISOCHI CHINWENDU AMORHA ${ }^{1^{* *}}$, CHIOMA LOVE OKPE ${ }^{2}$, OBINNA FELIX DIM $^{3}$
}

${ }^{1}$ Kosisochi Chinwendu Amorha (B. Pharm, M. Pharm, FPCPharm, Ph.D), ${ }^{2}$ Chioma Love Okpe (B. Pharm), ${ }^{3}$ Obinna Felix Dim (B. Pharm) Email: kosisochi.amorha@unn.edu.ng

Received: 18 May 2018 Revised and Accepted: 26 Sep 2018

\begin{abstract}
Objective: To assess the knowledge, attitudes and perceived practice of asthma among final year pharmacy students in Southern Nigeria.

Methods: This was a cross sectional study conducted in six pharmacy schools in Southern Nigeria. A 49-item structured, self-administered questionnaire was used to collect data from the final year pharmacy students. Data were analyzed using the IBM Statistical Product for Services Solution (SPSS) for Windows, Version 21.0. Both descriptive and inferential statistics were utilized. Statistical significance was set at $\mathrm{P}<0.05$.

Results: Overall, less than half of the students had good knowledge of asthma (47.7\%); good attitudes towards asthma (49.5\%); and good perception of the roles of pharmacists in counselling asthma patients (48.1\%). SIWES/IT (Students' Industrial Work Experience Scheme/Industrial Training) improved students' asthma knowledge $(\mathrm{t}=3.119 ; \mathrm{df}=453 ; \mathrm{P}=0.002)$. The University of Lagos (UNILAG) pharmacy students had significantly better asthma knowledge than their University of Uyo (UNIUYO), University of Nigeria Nsukka (UNN), Obafemi Awolowo University $(\mathrm{OAU})$ and University of Port Harcourt (UNIPORT) counterparts $(\mathrm{P}<0.05)$.

Conclusion: Less than half of the pharmacy students had good knowledge of asthma; good attitudes towards asthma and good perception of the roles of pharmacists in counselling asthma patients. UNILAG pharmacy students had significantly better asthma knowledge than most of the other schools. Students who had SIWES/IT experience (Students' Industrial Working Experience Scheme/Industrial Training) had better asthma knowledge. The SIWES/IT programme should continue.
\end{abstract}

Keywords: Attitudes, Asthma, Knowledge, Pharmacy Students, Practice

(C) 2018 The Authors. Published by Innovare Academic Sciences Pvt Ltd. This is an open access article under the CC BY license (http://creativecommons.org/licenses/by/4.0/] DOI: http://dx.doi.org/10.22159/ijpps.2018v10i11.27404

\section{INTRODUCTION}

The World Health Organization (WHO) recognizes asthma as a major health problem [1]. It is one of the common chronic diseases among children and is characterized by recurrent attacks of wheezing, breathlessness, cough, bronchial hyper-responsiveness and/or chest tightness, which vary in severity and frequency from person to person [2]. It can occur at any age but children and young adults are the commonly affected age groups. Environmental factors appear to be strongly associated with asthma in school children in a developing nation [3]. Both sexes are affected almost equally though slight differences in prevalence between males and females have been reported [4]. Although asthma cannot be "cured," clinical episodes can largely be prevented and controlled by proper management. Asthma is the leading cause of ambulatory visits and hospitalizations among children [4]. Asthma knowledge, health service quality, medical staff's attitude and skills, as well as patients' attitude and lifestyle determine the level of asthma control [5].

In Nigeria, the prevalence of asthma ranges from $10.7 \%$ (children), $14.2 \%$ (adolescents), and 5.1 to $7.5 \%$ (adults) in the general population $[6,7]$. Previous studies have shown that insufficient knowledge of asthma has been associated with inadequate disease management and increased morbidity [6]. Pharmacy students may have apathy towards practice in asthma settings if they do not have sufficient knowledge of asthma. Pharmacists have demonstrated poor knowledge of inhaler techniques and have not taken active roles in promoting self-management among asthma patients [5]. However, there is paucity of data on the knowledge, attitudes and perceived practice of pharmacy students towards asthma worldwide, Nigeria inclusive. The general objective of this study was to assess the knowledge, attitudes and perceived practice towards asthma among pharmacy students in Southern Nigeria.

\section{MATERIALS AND METHODS}

\section{Study design and study site}

This study was a descriptive, cross-sectional survey conducted in six pharmacy schools in Southern Nigeria to assess pharmacy students' knowledge, attitudes and perceived practice towards asthma. Southern Nigeria comprises three zones: South-west, South-south and South-east. Two schools were selected from each zone. The University of Nigeria, Nsukka, Enugu State (UNN) and Nnamdi Azikiwe University, Awka, Anambra State (NAU) from the Southeast; University of Port Harcourt (UNIPORT) and University of Uyo, Akwa Ibom State (UNIUYO); University of Lagos, Lagos State (UNILAG) and Obafemi Awolowo University, Ile-Ife, Osun State (OAU) from the South-west.

The aforementioned schools are all Federal Government institutions, accredited by the Nigerian Universities Commission (NUC) and the Pharmacists' Council of Nigeria (PCN).

\section{Ethical committee approval}

Ethical approval was obtained from the Health Research and Ethics Committee of the University of Nigeria Teaching Hospital (UNTH), Ituku-Ozalla, Enugu State (NHREC/05/01/2008B-FWA000024581RB00002323). The Consent of the Deans of the different Schools of Pharmacy was obtained before the study commenced.

\section{Eligibility criteria}

All final year pharmacy students in the six aforementioned pharmacy schools (UNN, NAU, UNIPORT, UNIUYO, UNILAG, OAU) who were willing to participate in the study were included.

\section{Data collection}

A pilot study was conducted with ten final year students of UNN and they were not included in the main study. Their responses were used 
to modify the questionnaire, ensuring there were no ambiguities in the statements/questions. All irrelevances were excluded. The final study instrument was a 49-item structured self-administered questionnaire divided into four sections: demographic information; knowledge on asthma; attitudes towards asthma; and perceived practice regarding asthma. Willingness to participate was taken as consent. The respondents were expected to fill the questionnaire within $15 \mathrm{~min}$, without the consultation of any reference material.

\section{Data analysis}

Data were analysed using the IBM Statistical Products and Service Solutions (SPSS) for Windows, Version 21.0 (IBM Corp, Version 21.0, Armonk, NY, USA). Descriptive statistics, such as mean \pm standard deviation, were used to summarize data. Inferential statistics utilized the independent t-test, ANOVA and Pearson Chi-Square test, with statistical significance set at $\mathrm{P} \leq 0.05$.

\section{RESULTS}

A total of 455 questionnaires administered were completed and returned from the six universities utilized for the study, representing an overall participation rate of $60.6 \%$ (455/751). For the individual universities, the participation rate was $94.8 \%(55 / 58)$ for the University of Uyo (UNIUYO); 70\% (63/90) for Nnamdi Azikiwe University (NAU); $47.9 \%$ (103/215) for University of Nigeria Nsukka (UNN); $56.5 \%(96 / 170)$ for the University of Lagos (UNILAG); 51.8\% (71/137) for Obafemi Awolowo University (OAU); $82.7 \%(67 / 81)$ for the University of Port Harcourt (UNIPORT). The results are presented in the tables below.

Most of the respondents (74.7\%) were between 21 to $25 \mathrm{y}$, had previous IT experience $(77.8 \%)$ in hospital/community pharmacy settings (72.7\%). See table 1 for more details.

Only $23.5 \%$ of the students got the question on short-acting $\beta_{2}$ agonists. Majority of them $(86.4 \%)$ knew that epinephrine has nothing to do with inflammatory reaction, table 2 . Overall, less than half (47.7\%) of the students had good knowledge of asthma.

Less than half of the students (48.3\%) disagreed that asthmatic patients may not benefit from disease monitoring with peak flow meter. Most of the students (93.7\%) agreed that pharmacist play an important role in the asthma care team, table 3. Overall, less than half $(49.5 \%)$ of the students had good attitudes towards asthma.

More than half of the students (86.8\%) agreed that the role of pharmacists include counselling patients about inhaler techniques on a regular basis. Also, most of the students (87.2\%) agreed that the role of pharmacist include counselling patients about asthma selfmanagement, table 4. Overall, less than half of the students (48.1\%) had good perception of the roles of pharmacists in counselling asthma patients.

More than half of the students considered that lack of time by pharmacists $(84.8 \%)$, patients' perception that they are already well cared for by the doctor $(81.5 \%)$, and lack of confidence or skill in asthma medication counselling (83.1\%) have considerable to high impact as barriers to providing specific asthma counselling or service. See table 5. Overall, more than half of the students (53.4\%) demonstrated that the items had low impact on pharmacists' ability to provide specific asthma counselling or service.

Almost all the students (93.8\%) agreed that they would have good professional contact with other healthcare professionals with regards to caring for patients with asthma, table 6 . Overall, $59.6 \%$ of the students believed they would have good inter-professional contact with regards to caring for patients with asthma.

In table 7, more female pharmacy students had better perceived practice regarding the role of pharmacists in counselling asthma patients $(\mathrm{t}=-$ 3.199; $\mathrm{df}=453 ; \mathrm{p}=0.001$ ) and were more predisposed to having interprofessional contact with other health care professionals in caring for asthma patients $(\mathrm{t}=-2.031 ; \mathrm{df}=453 ; \mathrm{P}=0.043)$, than males.

Also, more female pharmacy students had a higher perception of the barriers to pharmacists' abilities to provide specific asthma counselling or service $(\mathrm{t}=-2.921 ; \mathrm{df}=453 ; \mathrm{P}<0.004)$.

Students who resided in hostels had better asthma knowledge than those who stayed off-campus $(\mathrm{t}=3.771 ; \mathrm{df}=453 ; \mathrm{P}<0.001)$. Those who were not asthmatic had better attitudes towards asthma than those who had been diagnosed of asthma $(t=-4.176 ; F=453 ; \mathrm{P}<0.001)$.

Table 1: Socio-demographic information

\begin{tabular}{ll}
\hline Variables & n (\%) \\
\hline University & $55(12.1)$ \\
UNIUYO & $63(13.8)$ \\
NAU & $103(22.6)$ \\
UNN & $96(21.1)$ \\
UNILAG & $71(15.6)$ \\
OAU & $67(14.7)$ \\
UNIPORT & $23(5.1)$ \\
Age (in years) & $340(74.7)$ \\
$16-20$ & $70(15.4)$ \\
$21-25$ & $22(4.8)$ \\
$26-30$ & \\
$>30$ & $256(56.3)$ \\
Gender & $199(43.7)$ \\
Male & \\
Female & $398(87.5)$ \\
Religion & $49(10.8)$ \\
Christianity & $8(1.8)$ \\
Islam & \\
Others & $185(40.7)$ \\
Residency & $270(59.3)$ \\
Hostel campus & $42(9.2)$ \\
Diagnosed of asthma & $102(22.4)$ \\
Family history of asthma & $354(77.8)$ \\
Previous SIWES/IT experience & $331(72.7)$ \\
\hline Hospital/Community pharmacy IT &
\end{tabular}

SIWES = Students' Industrial Working Experience Scheme; IT = Industrial Training 
Those who had undergone SIWES/IT (Students Industrial Work Experience Scheme) ( $\mathrm{t}=3.119 ; \mathrm{df}=453 ; \mathrm{P}=0.002)$, especially in hospital/community pharmacy settings $(\mathrm{t}=4.109 ; \mathrm{df}=453 ; \mathrm{P}<0.001)$, had better knowledge of asthma than those who had not. There was statistically significant difference $(F=3.570)$ between the mean attitude scores for the age groups. The Post-Hoc multiple comparisons with Scheffe between the mean attitude scores showed a difference between students aged $16-20$ y and those between 21-25years $(\mathrm{P}=0.046)$ and $26-30$ years $(P=0.029)$. Since higher scores on attitudes indicate more positive attitudes towards asthma, students between 21-25 y and 26-30 y had significantly better attitudes than those between 16-20 y. Additionally, there was a statistically significant difference $(F=13.191)$ between the mean knowledge scores for university.

The Post-Hoc multiple comparisons with Scheffe showed differences between:

NAU and UNIUYO $(\mathrm{P}=0.020)$

UNILAG and UNIUYO $(\mathrm{P}<0.001)$

UNILAG and UNN (P = 0.018)

UNILAG and OAU $(\mathrm{P}<0.001)$

\section{UNILAG and UNIPORT $(\mathrm{p}<0.001)$}

Since higher scores on knowledge indicate better knowledge about asthma, NAU pharmacy students had significant better asthma knowledge than their UNIUYO counterparts. UNILAG pharmacy students had significant better asthma knowledge than UNIUYO, UNN, OAU and UNIPORT pharmacy students.

There was a statistically significant difference $(F=10.821)$ between the mean attitude scores for university. The post-Hoc multiple comparisons with Scheffe showed differences between:

UYO and UNILAG $(\mathrm{P}=0.023)$

NAU and UNILAG $(\mathrm{P}=0.002)$

UNN and UNILAG $(\mathrm{P}<0.001)$

OAU and UNILAG $(\mathrm{P}<0.001)$

UNIPORT and UNILAG $(\mathrm{P}=0.001)$

Since higher scores on attitudes indicates attitudes towards asthma: UNIUYO, NAU, UNN, OAU and UNIPORT students had statistically significant better attitudes than UNILAG students.

\section{Table 2: Knowledge of asthma}

\begin{tabular}{|c|c|}
\hline Questions (Correct answer) & $\mathrm{n}(\%)$ \\
\hline 1. Among the following physiological factors, which could affect theophylline blood concentration the most? (All of the above) & $262(57.6)$ \\
\hline 2. Which of the following could be a side effect of inhaled steroids? (All of the above) & $190(41.8)$ \\
\hline 3. Which of the following ranges characterizes the 'yellow zone' for peak flow meter measurements? $(60-80 \%)$ & $229(50.3)$ \\
\hline 4. Poor asthma control may be related to: (All of the above) & $388(85.3)$ \\
\hline 5. Which of the following is considered the cell that triggers an allergic reaction after exposure to the allergens? (Mast cell) & $342(75.2)$ \\
\hline $\begin{array}{l}\text { 6. What is the best time to use a peak flow meter, if the patient can use it only once daily? (After waking up, before going out in the } \\
\text { morning) }\end{array}$ & $213(46.8)$ \\
\hline 7. Which of the following has nothing to do with an inflammation reaction? (Epinephrine) & $393(86.4)$ \\
\hline $\begin{array}{l}\text { 8. Which one of the following is not a sympathomimetic drug, administered as aerosol, and could dilate the bronchioles with fewer } \\
\text { systemic side effects? (Ipratropium bromide). }\end{array}$ & $133(29.2)$ \\
\hline $\begin{array}{l}\text { 9. Long-term asthma control for children with moderate continuous asthma should include which of the following drugs? } \\
\text { (Beclomethasone, salmeterol and albuterol) }\end{array}$ & $174(38.2)$ \\
\hline 10. Which statement is true about short-acting $\beta_{2}$-agonists? (All of the above) & 107 (23.5) \\
\hline
\end{tabular}

Table 3: Attitudes towards asthma

\begin{tabular}{lllllll}
\hline Statements & SD & D & N & A & SA & Mean (SDv) \\
\hline $\begin{array}{l}\text { 1. Healthcare providers should understand the } \\
\text { influence of asthma care activities on patients' } \\
\text { daily lives }\end{array}$ & $0(0.0)$ & $0(0.0)$ & $9(2.0)$ & $96(21.1)$ & $350(76.9)$ & $4.75(0.48)$ \\
$\begin{array}{l}\text { 2. Pharmacists should be certified to provide } \\
\text { primary asthma care }\end{array}$ & $1(0.2)$ & $8(1.8)$ & $25(5.5)$ & $133(29.2)$ & $288(63.3)$ & $4.54(0.70)$ \\
$\begin{array}{l}\text { 3. The impact of asthma on patients' emotions is } \\
\text { minor }\end{array}$ & $94(20.7)$ & $137(30.1)$ & $87(19.1)$ & $81(17.8)$ & $56(12.3)$ & $3.29(1.31)$ \\
$\begin{array}{l}\text { 4. To become a competent asthma educator, it is } \\
\text { necessary to learn education skills }\end{array}$ & $3(0.7)$ & $17(3.7)$ & $46(10.1)$ & $174(38.2)$ & $215(47.3)$ & $4.28(0.84$ \\
$\begin{array}{l}\text { 5. The outcome of asthma treatment depends } \\
\text { more on a patient's behaviour than efforts of } \\
\text { healthcare providers }\end{array}$ & $10(2.2)$ & $41(9.0)$ & $87(19.1)$ & $182(40.0)$ & $135(29.7)$ & $2.14(1.01)$ \\
$\begin{array}{l}\text { 6. Asthmatic patients may not benefit from } \\
\text { disease monitoring with peak flow meter }\end{array}$ & $88(19.3)$ & $132(29.0)$ & $103(22.6)$ & $83(18.2)$ & $49(10.8)$ & $3.28(1.27)$ \\
$\begin{array}{l}\text { 7. Control of respiration function is too } \\
\text { complicated in asthma care }\end{array}$ & $29(6.4)$ & $100(22.0)$ & $125(27.5)$ & $136(29.9)$ & $65(14.3)$ & $2.76(1.14)$ \\
$\begin{array}{l}\text { 8. People with asthma have the right to decide } \\
\text { not to take care of their disease }\end{array}$ & $74(16.3)$ & $89(19.6)$ & $97(21.3)$ & $116(25.5)$ & $79(17.4)$ & $2.92(1.34)$ \\
$\begin{array}{l}\text { 9. The asthmatic patient is the most important } \\
\text { member in the asthma care team }\end{array}$ & $6(1.3)$ & $20(4.4)$ & $45(9.9)$ & $146(32.1)$ & $238(52.3)$ & $4.30(0.91)$ \\
$\begin{array}{l}\text { 10. The pharmacist could play an important role } \\
\text { in the asthma care team }\end{array}$ & $5(1.1)$ & $2(0.4)$ & $22(4.8)$ & $110(24.2)$ & $316(69.5)$ & $4.68(0.93)$ \\
\hline
\end{tabular}


Table 4: Perceived roles of pharmacists in counselling asthma patients

\begin{tabular}{|c|c|c|c|c|c|c|}
\hline Statements & SD & D & $\mathbf{N}$ & $\mathbf{A}$ & SA & Mean (SDv) \\
\hline 1. Frequency of reliever (blue) inhaler use. & $0(0.0)$ & $2(0.4)$ & $29(6.4)$ & $136(29.9)$ & $288(63.3)$ & $4.56(0.63)$ \\
\hline 2. Overuse of reliever (blue) medication & $5(1.1)$ & $18(4.0)$ & $35(7.7)$ & $153(33.6)$ & $244(53.6)$ & $4.35(0.87)$ \\
\hline 3. Poor adherence with preventer medication & $8(1.8)$ & $11(2.4)$ & $36(7.9)$ & $140(30.8)$ & $260(57.1)$ & $4.39(0.87)$ \\
\hline 4. Inhaler technique when first prescribed the inhaler & $0(0.0)$ & $5(1.1)$ & $43(9.5)$ & $114(25.1)$ & $293(64.4)$ & $4.53(0.71)$ \\
\hline 5. Inhaler technique on a regular basis. & $1(0.2)$ & $12(2.6)$ & $47(10.3)$ & $167(36.7)$ & $228(50.1)$ & $4.34(0.79)$ \\
\hline 6. Trigger factors and avoidance strategies & $2(0.4)$ & $4(0.9)$ & $41(9.0)$ & $125(27.5)$ & $283(62.2)$ & $4.50(0.73)$ \\
\hline 7. Patient's current level of asthma control & $0(0.0)$ & $0(0.0)$ & $54(11.9)$ & $170(37.4)$ & $231(50.8)$ & $4.39(0.69)$ \\
\hline 8. Action plan ownership & $1(0.2)$ & $9(2.0)$ & $73(16.0)$ & $166(36.5)$ & $206(45.3)$ & $4.25(0.81)$ \\
\hline $\begin{array}{l}\text { 9. Patient self-monitoring of asthma control (by symptoms or } \\
\text { peak flow measurements) }\end{array}$ & $4(0.9)$ & $12(2.6)$ & $42(9.2)$ & $159(34.9)$ & $238(52.3)$ & $4.35(0.82)$ \\
\hline $\begin{array}{l}\text { 10. Asthma self-management by the patient (i.e. recognizing } \\
\text { when and knowing how to take action when asthma gets worse) }\end{array}$ & $3(0.7)$ & $9(2.0)$ & $44(9.7)$ & $143(31.4)$ & $256(56.3)$ & $4.41(0.8)$ \\
\hline
\end{tabular}

SD-Strongly Disagree; D-Disagree; N-Neutral; A-agree; SA-Strongly Agree; SDv-Standard Deviation

Table 5: Perceived practice barriers to pharmacist's ability to provide specific asthma counselling or service

\begin{tabular}{|c|c|c|c|c|c|c|}
\hline Statements & No impact & $\begin{array}{l}\text { Slight } \\
\text { impact }\end{array}$ & $\begin{array}{l}\text { Moderate } \\
\text { impact }\end{array}$ & $\begin{array}{l}\text { Considerable } \\
\text { impact }\end{array}$ & $\begin{array}{l}\text { High } \\
\text { impact }\end{array}$ & Mean(SDv) \\
\hline 11. Lack of time by the pharmacist & $5(11)$ & $10(2.2)$ & $54(11.9)$ & $149(32.7)$ & $237(52.1)$ & $4.33(0.85)$ \\
\hline 12. Lack of time by the patient & $6(1.3)$ & $22(4.8)$ & $57(12.5)$ & $141(31.0)$ & $229(50.3)$ & $4.24(0.94)$ \\
\hline 13. Pharmacists' perception that it is not their role & $10(2.2)$ & $21(4.6)$ & $64(14.1)$ & $162(35.6)$ & $198(43.5)$ & $4.14(0.97)$ \\
\hline $\begin{array}{l}\text { 14. Patient's perception that it is not the pharmacist's } \\
\text { role }\end{array}$ & $9(2.0)$ & $23(5.1)$ & $72(15.8)$ & $154(33.8)$ & $197(43.3)$ & $4.11(0.9)$ \\
\hline 15. Language barriers & $4(0.9)$ & $22(4.8)$ & $48(10.5)$ & $152(33.4)$ & $229(50.3)$ & $4.27(0.90)$ \\
\hline 16. Patient's health beliefs & $0(0.0)$ & $16(3.5)$ & $61(13.4)$ & $153(33.6)$ & $225(49.5)$ & $4.29(0.83)$ \\
\hline 17. Patient's lack of asthma knowledge & $5(1.1)$ & $20(4.4)$ & $62(13.6)$ & $137(30.1)$ & $231(50.8)$ & $4.25(0.93)$ \\
\hline $\begin{array}{l}\text { 18. Patient perception that they are already well cared } \\
\text { for by the doctor }\end{array}$ & $3(0.7)$ & $19(4.2)$ & $62(13.6)$ & $167(36.7)$ & $204(44.8)$ & $4.21(0.88)$ \\
\hline $\begin{array}{l}\text { 19. Conflict between professional and commercial } \\
\text { interests }\end{array}$ & $3(0.7)$ & $17(3.7)$ & $64(14.1)$ & $167(36.7)$ & $204(44.8)$ & $4.21(0.87)$ \\
\hline 20. Trying not to 'overstep' the role of the doctor & $7(1.5)$ & $20(4.4)$ & $77(16.9)$ & $177(38.9)$ & $174(38.2)$ & $4.08(0.93)$ \\
\hline 21. No financial incentive. & $13(2.9)$ & $34(7.5)$ & $78(17.1)$ & $142(31.2)$ & $188(41.3)$ & $4.01(1.07)$ \\
\hline \multicolumn{7}{|l|}{ Lack of confidence or skills in: } \\
\hline 22. asthma medication counseling & $6(1.3)$ & $16(3.5)$ & $55(12.1)$ & $130(28.6)$ & $248(54.5)$ & $4.31(0.91)$ \\
\hline 23. asthma adherence counseling & $8(1.8)$ & $16(3.5)$ & $50(11.0)$ & $133(29.2)$ & $248(54.5)$ & $4.31(0.93)$ \\
\hline 24. asthma self-management counseling & $5(1.1)$ & $22(4.8)$ & $52(11.4)$ & $135(29.7)$ & $241(53.0)$ & $4.29(0.93)$ \\
\hline 25. asthma trigger factor counseling & $5(1.1)$ & $18(4.0)$ & $51(11.2)$ & $136(29.9)$ & $245(53.8)$ & $4.31(0.90)$ \\
\hline 26. reviewing and counselling about asthma control & $2(0.4)$ & $19(4.2)$ & $52(11.4)$ & $150(33.0)$ & $232(51.0)$ & $4.30(0.86)$ \\
\hline 27. asthma monitoring & $4(0.9)$ & $16(3.5)$ & $54(11.9)$ & $129(28.4)$ & $252(55.4)$ & $4.34(0.88)$ \\
\hline
\end{tabular}

SDv-Standard Deviation

Table 6: Inter-professional contact

\begin{tabular}{|c|c|c|c|c|c|c|}
\hline Statements & SD & D & $\mathbf{N}$ & $\mathbf{A}$ & SA & Mean(SDv) \\
\hline $\begin{array}{l}\text { 28. I would have good inter-professional contact with other healthcare } \\
\text { professionals with regards to care of my patients with asthma }\end{array}$ & $0(0.0)$ & $7(1.5)$ & $21(4.6)$ & $101(22.2)$ & $326(71.6)$ & $4.64(0.65)$ \\
\hline $\begin{array}{l}\text { 29. I would like to "go the extra mile" with other healthcare } \\
\text { professionals with regards to the care of my patients with asthma }\end{array}$ & $3(0.7)$ & $8(1.8)$ & $39(8.6)$ & 115 (25.3) & 290 (63.7) & $4.50(0.78)$ \\
\hline
\end{tabular}

SD-Strongly Disagree; D-Disagree; N-Neutral; A-agree; SA-Strongly Agree; SDv-Standard Deviation

Table 7a: Mean difference analysis (I)

\begin{tabular}{|c|c|c|c|c|c|c|c|c|c|c|c|c|c|c|c|c|}
\hline $\begin{array}{l}\text { Variab } \\
\text { les }\end{array}$ & $\begin{array}{l}\text { N } \\
\text { (tot } \\
\text { al } \\
=45 \\
5 \text { ) } \\
\end{array}$ & $\begin{array}{l}\text { Mean } \\
\text { knowl } \\
\text { edge } \\
\text { score } \\
\text { (SDv) }\end{array}$ & $\begin{array}{l}\mathbf{9 5 \%} \\
\text { confid } \\
\text { ence } \\
\text { interv } \\
\text { al }\end{array}$ & $\begin{array}{l}\text { p- } \\
\text { valu } \\
\text { e }\end{array}$ & $\begin{array}{l}\text { Mean } \\
\text { Attitu } \\
\text { de } \\
\text { score } \\
\text { (SDv) }\end{array}$ & $\begin{array}{l}95 \% \\
\text { confid } \\
\text { ence } \\
\text { interv } \\
\text { al }\end{array}$ & $\begin{array}{l}\text { P- } \\
\text { valu } \\
\text { e }\end{array}$ & $\begin{array}{l}\text { Mean } \\
\text { Roles } \\
\text { scores( } \\
\text { SDv) }\end{array}$ & $\begin{array}{l}95 \% \\
\text { confid } \\
\text { ence } \\
\text { interv } \\
\text { al }\end{array}$ & $\begin{array}{l}\text { P- } \\
\text { val } \\
\text { ue }\end{array}$ & $\begin{array}{l}\text { Mean } \\
\text { barrier } \\
\text { score(S } \\
\text { Dv) }\end{array}$ & $\begin{array}{l}95 \% \\
\text { confid } \\
\text { ence } \\
\text { interv } \\
\text { al }\end{array}$ & $\begin{array}{l}\text { P- } \\
\text { val } \\
\text { ue }\end{array}$ & $\begin{array}{l}\text { Mean } \\
\text { contac } \\
\text { t } \\
\text { score( } \\
\text { SDv) } \\
\end{array}$ & $\begin{array}{l}\mathbf{9 5 \%} \\
\text { confid } \\
\text { ence } \\
\text { interv } \\
\text { al }\end{array}$ & $\begin{array}{l}\text { P- } \\
\text { val } \\
\text { ue }\end{array}$ \\
\hline \multicolumn{17}{|l|}{$\begin{array}{l}\text { Age(in } \\
\text { years) } \\
\text { a }\end{array}$} \\
\hline $16-20$ & 23 & $\begin{array}{l}5.70 \\
(2.44)\end{array}$ & $\begin{array}{l}4.64- \\
6.75\end{array}$ & $\begin{array}{l}0.77 \\
5\end{array}$ & $\begin{array}{l}34.09 \\
(4.64)\end{array}$ & $\begin{array}{l}32.08- \\
36.09\end{array}$ & $\begin{array}{l}* 0.0 \\
14\end{array}$ & $\begin{array}{l}42.57 \\
(6.11)\end{array}$ & $\begin{array}{l}39.92- \\
45.21\end{array}$ & $\begin{array}{l}0.4 \\
96\end{array}$ & $\begin{array}{l}70.52 \\
(8.56)\end{array}$ & $\begin{array}{l}66.82- \\
74.23\end{array}$ & $\begin{array}{l}0.3 \\
97\end{array}$ & $\begin{array}{l}8.83 \\
(1.23)\end{array}$ & $\begin{array}{l}8.29- \\
9.36\end{array}$ & $\begin{array}{l}0.1 \\
44\end{array}$ \\
\hline $21-25$ & 340 & $\begin{array}{l}5.31 \\
(1.80)\end{array}$ & $\begin{array}{l}5.11- \\
5.50\end{array}$ & & $\begin{array}{l}36.98 \\
(4.63)\end{array}$ & $\begin{array}{l}36.49- \\
37.47\end{array}$ & & $\begin{array}{l}44.16 \\
(5.45)\end{array}$ & $\begin{array}{l}43.58- \\
44.74\end{array}$ & & $\begin{array}{l}72.26 \\
(10.27)\end{array}$ & $\begin{array}{l}71.16- \\
73.35\end{array}$ & & $\begin{array}{l}9.09 \\
(1.35)\end{array}$ & $\begin{array}{l}8.95- \\
9.23\end{array}$ & \\
\hline $26-30$ & 70 & $\begin{array}{l}5.37 \\
(1.64)\end{array}$ & $\begin{array}{l}4.98- \\
5.76\end{array}$ & & $\begin{array}{l}37.51 \\
(5.02)\end{array}$ & $\begin{array}{l}36.32- \\
38.71\end{array}$ & & $\begin{array}{l}43.80 \\
(6.06)\end{array}$ & $\begin{array}{l}42.36- \\
45.24\end{array}$ & & $\begin{array}{l}70.60 \\
(10.92)\end{array}$ & $\begin{array}{l}68.00- \\
73.20\end{array}$ & & $\begin{array}{l}9.33 \\
(1.19)\end{array}$ & $\begin{array}{l}9.05- \\
9.61\end{array}$ & \\
\hline$>30$ & 22 & $\begin{array}{l}5.45 \\
(1.70) \\
\end{array}$ & $\begin{array}{l}4.66- \\
6.25 \\
\end{array}$ & & $\begin{array}{l}35.73 \\
(5.43) \\
\end{array}$ & $\begin{array}{l}33.32- \\
38.13 \\
\end{array}$ & & $\begin{array}{l}44.86 \\
(4.99) \\
\end{array}$ & $\begin{array}{l}42.65- \\
47.08 \\
\end{array}$ & & $\begin{array}{l}74.09 \\
(6.54) \\
\end{array}$ & $\begin{array}{l}71.9- \\
76.99 \\
\end{array}$ & & $\begin{array}{l}9.55 \\
(0.86) \\
\end{array}$ & $\begin{array}{l}9.17- \\
9.93 \\
\end{array}$ & \\
\hline
\end{tabular}




\begin{tabular}{|c|c|c|c|c|c|c|c|c|c|c|c|c|c|c|c|c|}
\hline \multicolumn{17}{|l|}{$\begin{array}{l}\text { Gender } \\
\mathrm{b}\end{array}$} \\
\hline Male & 256 & $\begin{array}{l}5.26 \\
(1.77)\end{array}$ & $\begin{array}{l}-0.52- \\
0.15\end{array}$ & $\begin{array}{l}0.27 \\
9\end{array}$ & $\begin{array}{l}36.51( \\
4.81)\end{array}$ & $\begin{array}{l}-1.68- \\
0.09\end{array}$ & $\begin{array}{l}0.07 \\
8\end{array}$ & $\begin{array}{l}43.33 \\
(5.69)\end{array}$ & $\begin{array}{l}- \\
2.68- \\
0.64\end{array}$ & $\begin{array}{l}* 0.0 \\
01\end{array}$ & $\begin{array}{l}70.79 \\
(10.12)\end{array}$ & $\begin{array}{l}- \\
4.65- \\
0.91\end{array}$ & $\begin{array}{l}* 0.0 \\
04\end{array}$ & $\begin{array}{l}9.03 \\
(1.37)\end{array}$ & $\begin{array}{l}- \\
0.49- \\
0.008\end{array}$ & $\begin{array}{l}* 0.0 \\
43\end{array}$ \\
\hline Female & 199 & $\begin{array}{l}5.45 \\
(1.86)\end{array}$ & & & $\begin{array}{l}37.30 \\
(4.70)\end{array}$ & & & $\begin{array}{l}45.00 \\
(5.25)\end{array}$ & & & $\begin{array}{l}73.57 \\
(10.00)\end{array}$ & & & $\begin{array}{l}9.28 \\
(1.20)\end{array}$ & & \\
\hline \multicolumn{17}{|l|}{$\begin{array}{l}\text { Religio } \\
n \text { a }\end{array}$} \\
\hline $\begin{array}{l}\text { Christi } \\
\text { anity }\end{array}$ & 398 & $\begin{array}{l}5.32 \\
(1.76)\end{array}$ & $\begin{array}{l}5.15- \\
5.50\end{array}$ & $\begin{array}{l}0.37 \\
0\end{array}$ & $\begin{array}{l}37.10 \\
(4.74)\end{array}$ & $\begin{array}{l}36.63- \\
37.57\end{array}$ & $\begin{array}{l}0.01 \\
4\end{array}$ & $\begin{array}{l}44.16 \\
(5.54)\end{array}$ & $\begin{array}{l}43.61- \\
44.70\end{array}$ & $\begin{array}{l}0.5 \\
83\end{array}$ & $\begin{array}{l}71.88(1 \\
0.28)\end{array}$ & $\begin{array}{l}70.87- \\
72.89\end{array}$ & $\begin{array}{l}0.5 \\
59\end{array}$ & $\begin{array}{l}9.15 \\
(1.30)\end{array}$ & $\begin{array}{l}9.02- \\
9.28\end{array}$ & $\begin{array}{l}0.1 \\
89\end{array}$ \\
\hline Islam & 49 & $\begin{array}{l}5.61 \\
(2.19)\end{array}$ & $\begin{array}{l}4.98- \\
6.24\end{array}$ & & $\begin{array}{l}35.04 \\
(4.56)\end{array}$ & $\begin{array}{l}33.73- \\
36.35\end{array}$ & & $\begin{array}{l}43.45 \\
(5.91)\end{array}$ & $\begin{array}{l}41.75- \\
45.14\end{array}$ & & $\begin{array}{l}73.33 \\
(9.16)\end{array}$ & $\begin{array}{l}70.70- \\
75.96\end{array}$ & & $\begin{array}{l}9.04 \\
(1.40)\end{array}$ & $\begin{array}{l}8.64- \\
9.44\end{array}$ & \\
\hline Others & 8 & $\begin{array}{l}4.75 \\
(1.67)\end{array}$ & $\begin{array}{l}3.35- \\
6.15\end{array}$ & & $\begin{array}{l}35.75 \\
(5.52)\end{array}$ & $\begin{array}{l}31.13- \\
40.37\end{array}$ & & $\begin{array}{l}42.88 \\
(4.32)\end{array}$ & $\begin{array}{l}39.26- \\
46.49\end{array}$ & & $\begin{array}{l}70.13 \\
(9.52)\end{array}$ & $\begin{array}{l}62.16- \\
78.09\end{array}$ & & $\begin{array}{l}9.14 \\
(1.30)\end{array}$ & $\begin{array}{l}9.02- \\
9.26\end{array}$ & \\
\hline \multicolumn{17}{|l|}{$\begin{array}{l}\text { Reside } \\
\text { ncyb }\end{array}$} \\
\hline Hostel & 185 & $\begin{array}{l}5.72 \\
(1.83)\end{array}$ & $\begin{array}{l}0.31- \\
0.98\end{array}$ & $\begin{array}{l}*<0 \\
001\end{array}$ & $\begin{array}{l}36.45 \\
(5.12)\end{array}$ & $\begin{array}{l}-1.58- \\
0.21\end{array}$ & $\begin{array}{l}0.13 \\
3\end{array}$ & $\begin{array}{l}44.28 \\
(5.57)\end{array}$ & $\begin{array}{l}-0.67- \\
1.42\end{array}$ & $\begin{array}{l}0.4 \\
82\end{array}$ & $\begin{array}{l}72.66 \\
(9.65)\end{array}$ & $\begin{array}{l}-0.79- \\
3.02\end{array}$ & $\begin{array}{l}0.2 \\
51\end{array}$ & $\begin{array}{l}9.21 \\
(1.19)\end{array}$ & $\begin{array}{l}-0.13- \\
0.36\end{array}$ & $\begin{array}{l}0.3 \\
49\end{array}$ \\
\hline $\begin{array}{l}\text { Off- } \\
\text { campu } \\
\text { s } \\
\text { Diagno } \\
\text { sed of } \\
\text { asthma } \\
\text { b }\end{array}$ & 270 & $\begin{array}{l}5.08 \\
(1.75)\end{array}$ & & & $\begin{array}{l}37.13 \\
(4.51)\end{array}$ & & & $\begin{array}{l}43.91 \\
(5.55)\end{array}$ & & & $\begin{array}{l}71.55 \\
(10.47)\end{array}$ & & & $\begin{array}{l}9.09 \\
(1.37)\end{array}$ & & \\
\hline Yes & 42 & $\begin{array}{l}5.48(2 . \\
43)\end{array}$ & $\begin{array}{l}-0.43- \\
0.73\end{array}$ & $\begin{array}{l}0.61 \\
7\end{array}$ & $\begin{array}{l}33.98 \\
(5.16)\end{array}$ & $\begin{array}{l}-4.66- \\
1.68\end{array}$ & $\begin{array}{l}*<0 . \\
001\end{array}$ & $\begin{array}{l}44.71 \\
(4.48)\end{array}$ & $\begin{array}{l}-1.05- \\
2.49\end{array}$ & $\begin{array}{l}0.4 \\
23\end{array}$ & $\begin{array}{l}73.69 \\
(8.09)\end{array}$ & $\begin{array}{l}-1.37- \\
5.09\end{array}$ & $\begin{array}{l}0.2 \\
59\end{array}$ & $\begin{array}{l}9.14 \\
(1.20)\end{array}$ & $\begin{array}{l}-0.41- \\
0.42\end{array}$ & $\begin{array}{l}0.9 \\
73\end{array}$ \\
\hline No & 413 & $\begin{array}{l}5.33(1 . \\
74)\end{array}$ & & & $\begin{array}{l}37.15( \\
4.64)\end{array}$ & & & $\begin{array}{l}44.00 \\
(5.65)\end{array}$ & & & $\begin{array}{l}71.83 \\
(10.33)\end{array}$ & & & $\begin{array}{l}9.14 \\
(1.31) \\
\end{array}$ & & \\
\hline
\end{tabular}

Table 7b: Mean difference analysis (II)

\begin{tabular}{|c|c|c|c|c|c|c|c|c|c|c|c|c|c|c|c|c|}
\hline Variables & $\begin{array}{l}N \\
\text { (to } \\
\text { tal } \\
=4 \\
55 \text { ) } \\
\end{array}$ & $\begin{array}{l}\text { Mean } \\
\text { knowl } \\
\text { edge } \\
\text { score } \\
\text { (SDv) } \\
\end{array}$ & $\begin{array}{l}95 \% \\
\text { confid } \\
\text { ence } \\
\text { interv } \\
\text { al } \\
\end{array}$ & $\begin{array}{l}\text { p- } \\
\text { valu } \\
\text { e }\end{array}$ & $\begin{array}{l}\text { Mean } \\
\text { Attitu } \\
\text { de } \\
\text { score( } \\
\text { SDv) } \\
\end{array}$ & $\begin{array}{l}95 \% \\
\text { confid } \\
\text { ence } \\
\text { interv } \\
\text { al } \\
\end{array}$ & $\begin{array}{l}\text { P- } \\
\text { valu } \\
\text { e }\end{array}$ & $\begin{array}{l}\text { Mean } \\
\text { Roles } \\
\text { scores } \\
\text { (SDv) }\end{array}$ & $\begin{array}{l}95 \% \\
\text { confid } \\
\text { ence } \\
\text { interv } \\
\text { al } \\
\end{array}$ & $\begin{array}{l}\text { P- } \\
\text { val } \\
\text { ue }\end{array}$ & $\begin{array}{l}\text { Mean } \\
\text { barrie } \\
\mathbf{r} \\
\text { score( } \\
\text { SDv) } \\
\end{array}$ & $\begin{array}{l}\text { 95\%conf } \\
\text { idence } \\
\text { interval }\end{array}$ & $\begin{array}{l}\text { P- } \\
\text { val } \\
\text { ue }\end{array}$ & $\begin{array}{l}\text { Mean } \\
\text { contac } \\
\text { t } \\
\text { score( } \\
\text { SDv) } \\
\end{array}$ & $\begin{array}{l}95 \% \\
\text { confid } \\
\text { ence } \\
\text { interv } \\
\text { al } \\
\end{array}$ & $\begin{array}{l}\text { P- } \\
\text { val } \\
\text { ue }\end{array}$ \\
\hline \multicolumn{17}{|l|}{$\begin{array}{l}\text { Family } \\
\text { history of } \\
\text { asthma }^{b}\end{array}$} \\
\hline Yes & $\begin{array}{l}10 \\
2\end{array}$ & $\begin{array}{l}5.29 \\
(2.02)\end{array}$ & $\begin{array}{l}-0.46- \\
0.34\end{array}$ & $\begin{array}{l}0.75 \\
8\end{array}$ & $\begin{array}{l}36.28 \\
(4.76)\end{array}$ & $\begin{array}{l}-1.79- \\
0.32\end{array}$ & $\begin{array}{l}0.17 \\
1\end{array}$ & $\begin{array}{l}44.29 \\
(5.47)\end{array}$ & $\begin{array}{l}-0.93- \\
1.53\end{array}$ & $\begin{array}{l}0.6 \\
29\end{array}$ & $\begin{array}{l}72.47 \\
(9.06)\end{array}$ & $\begin{array}{l}-1.64- \\
2.84\end{array}$ & $\begin{array}{l}0.5 \\
99\end{array}$ & $\begin{array}{l}9.12 \\
(1.24)\end{array}$ & $\begin{array}{l}-0.31- \\
0.26\end{array}$ & $\begin{array}{l}0.8 \\
70\end{array}$ \\
\hline No & $\begin{array}{l}35 \\
3\end{array}$ & $\begin{array}{l}5.36 \\
(1.75)\end{array}$ & & & $\begin{array}{l}37.02 \\
(4.77)\end{array}$ & & & $\begin{array}{l}43.99 \\
(5.58)\end{array}$ & $\begin{array}{l}-0.92- \\
1.52\end{array}$ & & $\begin{array}{l}71.87 \\
(10.45)\end{array}$ & $\begin{array}{l}-1.48- \\
2.68\end{array}$ & & $\begin{array}{l}9.14 \\
(1.32)\end{array}$ & $\begin{array}{l}-0.30- \\
0.25\end{array}$ & \\
\hline \multicolumn{17}{|l|}{$\begin{array}{l}\text { Previous IT } \\
\text { experience }\end{array}$} \\
\hline Yes & $\begin{array}{l}35 \\
4\end{array}$ & $\begin{array}{l}5.48 \\
(1.80)\end{array}$ & $\begin{array}{l}0.23- \\
1.03\end{array}$ & $\begin{array}{l}* 0.0 \\
02\end{array}$ & $\begin{array}{l}36.79 \\
(4.83)\end{array}$ & $\begin{array}{l}-1.37- \\
0.75\end{array}$ & $\begin{array}{l}0.56 \\
1\end{array}$ & $\begin{array}{l}44.23 \\
(5.55)\end{array}$ & $\begin{array}{l}-0.48- \\
1.98\end{array}$ & $\begin{array}{l}0.2 \\
31\end{array}$ & $\begin{array}{l}71.99 \\
(10.13)\end{array}$ & $\begin{array}{l}-2.30- \\
2.21\end{array}$ & $\begin{array}{l}0.9 \\
69\end{array}$ & $\begin{array}{l}9.14 \\
(1.36)\end{array}$ & $\begin{array}{l}-0.29- \\
0.29\end{array}$ & $\begin{array}{l}0.9 \\
84\end{array}$ \\
\hline No & $\begin{array}{l}10 \\
1\end{array}$ & $\begin{array}{l}4.85 \\
(1.78)\end{array}$ & & & $\begin{array}{l}37.10 \\
(4.58)\end{array}$ & & & $\begin{array}{l}43.48 \\
(5.56)\end{array}$ & & & $\begin{array}{l}72.04 \\
(10.26)\end{array}$ & & & $\begin{array}{l}9.14 \\
(1.10)\end{array}$ & & \\
\hline \multicolumn{17}{|l|}{$\begin{array}{l}\text { IT in } \\
\text { hospital/Co } \\
\text { mmunity } \\
\text { pharmacyb }\end{array}$} \\
\hline Yes & $\begin{array}{l}33 \\
1\end{array}$ & $\begin{array}{l}5.55 \\
(1.71)\end{array}$ & $\begin{array}{l}0.40- \\
1.14\end{array}$ & $\begin{array}{l}<0.0 \\
01\end{array}$ & $\begin{array}{l}36.84( \\
4.84)\end{array}$ & $\begin{array}{l}-1.06- \\
0.92\end{array}$ & $\begin{array}{l}0.89 \\
5\end{array}$ & $\begin{array}{l}44.34( \\
5.59)\end{array}$ & $\begin{array}{l}-0.13- \\
2.16\end{array}$ & $\begin{array}{l}0.0 \\
83\end{array}$ & $\begin{array}{l}72.24( \\
10.16)\end{array}$ & $\begin{array}{l}-1.23- \\
2.97\end{array}$ & $\begin{array}{l}0.4 \\
16\end{array}$ & $\begin{array}{l}9.19(1 . \\
31)\end{array}$ & $\begin{array}{l}-0.08- \\
0.46\end{array}$ & $\begin{array}{l}0.1 \\
72\end{array}$ \\
\hline No & $\begin{array}{l}12 \\
4\end{array}$ & $\begin{array}{l}4.78(1 . \\
95)\end{array}$ & & & $\begin{array}{l}36.90( \\
4.60)\end{array}$ & & & $\begin{array}{l}43.32( \\
5.43)\end{array}$ & & & $\begin{array}{l}71.37( \\
10.13)\end{array}$ & & & $\begin{array}{l}9.00(1 . \\
28)\end{array}$ & & \\
\hline \multicolumn{17}{|l|}{ Universitya } \\
\hline UYO & 55 & $\begin{array}{l}4.51 \\
(1.67)\end{array}$ & $\begin{array}{l}4.06- \\
4.96\end{array}$ & $\begin{array}{l}*<0 \\
001\end{array}$ & $\begin{array}{l}36.80 \\
(4.18)\end{array}$ & $\begin{array}{l}35.67- \\
37.93\end{array}$ & $\begin{array}{l}*<0 \\
001\end{array}$ & $\begin{array}{l}43.71 \\
(6.04)\end{array}$ & $\begin{array}{l}42.08- \\
45.34\end{array}$ & $\begin{array}{l}0.1 \\
33\end{array}$ & $\begin{array}{l}69.22 \\
(12.25)\end{array}$ & $\begin{array}{l}65.91- \\
72.53\end{array}$ & $\begin{array}{l}0.2 \\
75\end{array}$ & $\begin{array}{l}9.22 \\
(1.33)\end{array}$ & $\begin{array}{l}8.86- \\
9.58\end{array}$ & $\begin{array}{l}0.8 \\
81\end{array}$ \\
\hline NAU & 63 & $\begin{array}{l}5.67 \\
(1.44)\end{array}$ & $\begin{array}{l}5.30- \\
6.03\end{array}$ & $\begin{array}{l}*<0 \\
001\end{array}$ & $\begin{array}{l}37.29 \\
(4.15)\end{array}$ & $\begin{array}{l}36.24- \\
38.33\end{array}$ & & $\begin{array}{l}45.67 \\
(3.99)\end{array}$ & $\begin{array}{l}44.66- \\
46.67\end{array}$ & & $\begin{array}{l}73.23 \\
(10.37)\end{array}$ & $\begin{array}{l}70.50- \\
75.98\end{array}$ & & $\begin{array}{l}9.22 \\
(1.33)\end{array}$ & $\begin{array}{l}8.86- \\
9.58\end{array}$ & \\
\hline UNN & $\begin{array}{l}10 \\
3\end{array}$ & $\begin{array}{l}5.45 \\
(1.64)\end{array}$ & $\begin{array}{l}5.13- \\
5.77\end{array}$ & & $\begin{array}{l}38.50 \\
(5.04)\end{array}$ & $\begin{array}{l}37.51- \\
39.48\end{array}$ & & $\begin{array}{l}44.28 \\
(6.40)\end{array}$ & $\begin{array}{l}43.03- \\
45.53\end{array}$ & & $\begin{array}{l}71.54 \\
(10.14)\end{array}$ & $\begin{array}{l}69.51- \\
73.58\end{array}$ & & $\begin{array}{l}9.00(1 . \\
46)\end{array}$ & $\begin{array}{l}8.71- \\
9.29\end{array}$ & \\
\hline UNILAG & 96 & $\begin{array}{l}6.34 \\
(2.06)\end{array}$ & $\begin{array}{l}5.93- \\
6.76\end{array}$ & & $\begin{array}{l}34.01 \\
(7.48)\end{array}$ & $\begin{array}{l}33.10- \\
34.91\end{array}$ & & $\begin{array}{l}43.13 \\
(5.64)\end{array}$ & $\begin{array}{l}41.98- \\
44.27\end{array}$ & & $\begin{array}{l}72.85( \\
8.90)\end{array}$ & $\begin{array}{l}71.05- \\
74.66\end{array}$ & & $\begin{array}{l}9.17(1 . \\
28)\end{array}$ & $\begin{array}{l}8.91- \\
9.43\end{array}$ & \\
\hline OAU & 71 & $\begin{array}{l}4.83(1 . \\
53)\end{array}$ & $\begin{array}{l}4.47- \\
5.19\end{array}$ & & $\begin{array}{l}37.46( \\
4.78)\end{array}$ & $\begin{array}{l}36.33- \\
38.60\end{array}$ & & $\begin{array}{l}43.99 \\
(5.29)\end{array}$ & $\begin{array}{l}42.73- \\
45.24\end{array}$ & & $\begin{array}{l}72.73 \\
(10.15)\end{array}$ & $\begin{array}{l}70.33- \\
75.14\end{array}$ & & $\begin{array}{l}9.11(1 . \\
23)\end{array}$ & $\begin{array}{l}8.82- \\
9.40\end{array}$ & \\
\hline UNIPORT & 67 & $\begin{array}{l}4.67 \\
(1.66) \\
\end{array}$ & $\begin{array}{l}4.27- \\
5.08\end{array}$ & & $\begin{array}{l}37.40 \\
(4.13)\end{array}$ & $\begin{array}{l}36.40- \\
38.41 \\
\end{array}$ & & $\begin{array}{l}43.91 \\
(4.99)\end{array}$ & $\begin{array}{l}42.69- \\
45.13 \\
\end{array}$ & & $\begin{array}{l}71.85( \\
8.59)\end{array}$ & $\begin{array}{l}69.76- \\
73.95 \\
\end{array}$ & & $\begin{array}{l}9.18(1 . \\
24)\end{array}$ & $\begin{array}{l}8.88- \\
9.48 \\
\end{array}$ & \\
\hline
\end{tabular}

Tests: $\mathrm{a}=$ Anova; $\mathrm{b}=$ Independent $\mathrm{t}$-test; ${ }^{*} \mathrm{P}<0.05=$ is statistically significant; $\mathrm{SDv}=$ standard deviation

\section{DISCUSSION}

In this study, less than half of the students had good knowledge of asthma; good attitudes towards asthma; and good perception of the roles of pharmacists in counselling asthma patients. SIWES/IT (Students' Industrial Work Experience Scheme/Industrial Training) improved students' asthma knowledge. UNILAG pharmacy students had significantly better asthma knowledge than their UNIUYO, UNN, OAU and UNIPORT counterparts. Close to a quarter of the participants had family history of asthma. This depicts the prevalence of asthma in Nigeria which affects about 15 million people in the country [7]. Overall, less than half of the students had 
good knowledge of asthma. Most of the knowledge-based assessments were basics of asthma treatment, and clearly indicate the need for more comprehensive training of pharmacy students. This is particularly important, now that pharmacy practice is playing an increasingly greater role in healthcare, especially through the introduction of specialized ward pharmacy services in hospitals [8]. The remarkable gap in pharmacy knowledge suggests that students might require more exposure than provided by their undergraduate pharmacotherapy lecture on asthma. Thus, asthma education should be started early and taught with proper teaching aids and techniques, including dummy devices.

The Students' Industrial Work Experience Scheme (SIWES) experience improved students' knowledge of asthma, emphasising the importance of exposing students to practice. A study conducted in Taiwan buttressed the importance of continuing education among pharmacists, with the conclusion that continuing education improves knowledge of pharmacists and would lead to more effective patient education, improved patient knowledge, and encourage proper inhaler techniques [12]. Also, only about half of the students were able to identify the yellow zone for peak flow meter measurements. This could be dependent on the extent to which teaching aids and instruments are employed while teaching asthma in pharmacy schools. Students are more likely to be exposed to these in practice settings. Peak flow meter and spirometers are important for measuring lung function as they help to monitor the progression of the disease and patients' response to therapy. It is documented that the knowledge and practice of spirometry is poor among hospital-based Nigerian health care professionals [14].

In this study, less than half of the students showed good attitudes towards asthma. If pharmacists have good attitudes towards a disease state, it could influence their level of involvement with rendering patient-centred services, considering that in Nigeria, many pharmaceutical care services are not paid for $[9,10]$. The quality of pharmaceutical care rendered in asthma will depend on the combination of the right attitudes and knowledge base of the pathophysiology and pharmacotherapy of the disease [17]. As pharmaceutical care is patient-centred, pharmacists can encourage patients to be involved with self-management, consequently improving their adherence to long-term medication therapies [17]. In a study conducted at the University of Wyoming School of Pharmacy to show pharmacy students' application of their knowledge from the classroom to introductory pharmacy practice experiences, it was concluded that challenging students to think about and provide specific examples of knowledge application made the connections more explicit and also allowed students to see the relevance of the classroom portion of their education and reinforced how foundational course concepts are directly applicable to pharmacy practice [8].

Few published studies have explored pharmacists' perceived roles in asthma management and counselling. Research in this area has primarily focused on structured community pharmacy based asthma programmes $[15,16]$. Overall, less than half of the students had good perception of the roles of pharmacists in counselling asthma patients. These may stem from inadequate exposure to pharmacy practice as students, and could affect their counselling skills/competence when they eventually become pharmacists. This was clearly justified by the attitudes of pharmacy students who participated in the SIWES scheme. They scored better in their perceived roles as pharmacists involved with counselling asthma patients. The SIWES scheme is a skill development programme established by Industrial Training Fund (ITF) in 1973 with the headquarters in Jos, Nigeria. It is meant to enable students in tertiary institutions in Nigeria acquire technical skills and experience for professional development in their course of study as it bridges the gap between theory and practice [11]. It enables students practice what they learnt in classroom. In some pharmacy schools in Nigeria, it is not mandatory for pharmacy students to be involved with SIWES. Regularly counselling patients on inhaler techniques and self-management will help improve adherence to medications with the achievement of the desired therapeutic outcomes [13]. Pharmacy students can inculcate this practice by learning from their senior colleagues during the SIWES.
Most of the students believed that lack of time by both patients and pharmacists and confidence/skill in asthma medication counselling are the greatest impediments to achieving the desired therapeutic outcome. Patient counselling requires the pharmacist to allot sufficient time, accordingly. Factors such as large numbers of patients per pharmacist or the patient's eagerness for fast discharge constitute the major challenges that pharmacists face [15].

Furthermore, more than half of the students considered language barrier to have a high impact on pharmacist ability to deliver pharmaceutical service since Nigeria is a multi-ethnic group. A pharmacist who cannot speak the patient's language cannot efficiently communicate, and a mediator between the pharmacist and the patient may be necessary. Also, pharmacists should avoid the use of medical jargons but as much as possible strive to be understood by the patient [17]. Most of the students agreed that financial incentives have a low impact on the ability of pharmacist to provide specific asthma counselling or services since pharmacist's consultation fee is not common in this part of the world [15]. The respondents believed that patients' perception that they are already well taken care of by the doctor has a considerable to high impact on the ability of pharmacists to provide specific asthma counselling. Doctors may believe that it is the responsibility of pharmacists to counsel asthma patients on their medications, especially at the point-of-purchase $[14,16]$. The confusion on whose role it is to counsel may lead to the asthma patients not being counselled. Pharmacists should never dispense medications, including inhalers, without adequate counselling [16].

Our study had some limitations. The study was carried out when some pharmacy schools were having their final professional examinations which could have reduced their participation rate. Assessments were only made using self-reported data. Also, the study was conducted in Southern Nigeria and the results might not be generalized to other study settings.

We recommend that the Pharmacists' Council of Nigeria which is responsible for regulating education and training of pharmacy students should provide an education standard that provides connections between classroom coursework and practice. The SIWES programme should continue and be enshrined in the pharmacy school curriculum. Asthma lectures should be taught with teaching aids such as power-point presentations, videos, dummy inhalers and spacer devices, peak flow meters, which will improve understanding of the disease state and its management.

\section{CONCLUSION}

Less than half of the students had good knowledge of asthma; good attitudes towards asthma and good perception of the roles of pharmacists in counselling asthma patients. UNILAG pharmacy students had significantly better asthma knowledge than most of the other schools. Students who had SIWES/IT experience (Students' Industrial Working Experience Scheme/Industrial Training) had better asthma knowledge. The SIWES/IT programme should continue.

\section{ACKNOWLEDGEMENTS}

We thank Pharmacists Oluwatosin Caleb Adeyemi (UNILAG), Deborah Obehi Onwusah (UNIPORT), Sunday Odunke Nduka (NAU), Ekpedeme Essien Ndem (UNIUYO).

\section{AUTHOR'S CONTRIBUTIONS}

KCA conceived and designed the study and participated in the data analysis; CLO was involved with the data acquisition; OFD drafted the manuscript. All authors read the final version of the manuscript.

\section{CONFLICTS OF INTERESTS}

All authors have no conflicts of interest to declare

\section{REFERENCES}

1. Rakhee S, Rajendra P, Kushwaha R, Surya K, Verma S, Garg R. A study to know the knowledge, attitude, and practices of patients of bronchial asthma. Int J Med Sci Public Health 2013; 3:159-62. 
2. World Health Organization. WHO fact sheet on asthma. Sixtyfirst World Health Assembly; 2008. Available from: http://www.who.int/respiratory/asthma/en/. [Last accessed on 16 May 2018].

3. Prakruthi GM, Bharathi DR, Yogananda R. A case control study on determinants of childhood asthma in school children of Chitradurga city. Int J Curr Pharm Res 2018;10:11-5.

4. Ganapathy RS. A prospective clinical study on disease knowledge and medication adherence pattern among asthmatic patients in tertiary care hospital in a Tirupur population. Asian J Pharm Clin Res 2017;10:388.

5. Sari CP, Hakim L, Dewa P. Role of pharmacist in counseling asthma to improve patient adherence in Yogyakarta. Asian J Pharm Clin Res 2017;10:16-20.

6. Oni AO, Erhabor GE, Egbagbe EE. The prevalence, management and burden of asthma-a Nigerian study. Iran J Allergy Asthma Immunol 2010;9:35-41.

7. Ekpimah E. 100 million Nigerians suffer from asthma in 2025. Punch Newspaper; 2016. Available from: http://www. punchng.com/100-million-nigerians-may-suffer-asthma2025/. [Last accessed 12 Dec 2017]

8. Krueger JL. Pharmacy students' application of knowledge from the classroom to introductory pharmacy practice experiences. Am J Pharm Educ 2013;77:3-9.

9. Gibson PG, Henry RL, Vimpani GV, Halliday J. Asthma knowledge, attitudes, and quality of life in adolescents. Arch Dis Child 1995;73:321-6. Available from: http://www. ncbi.nlm.nih.gov/entrez/query.fcgi?cmd=Retrieveanddb=Pub Medanddopt=Citationandlist_uids $=7492196 \% 5$ Cnpapers2://p
ublication/uuid/E68BD9C6-68A8-468D-AEE8-

A1DBED11D681. [Last accessed 16 May 2018]

10. Prasad R, Kushwaha RAS, Verma S, Kumar S, Verma A. A study to know the knowledge, attitude, and practices of patients of bronchial asthma. Int J Med Sci Public Health 2013;3:159.

11. Chukwuedo S. Students perception of the influence of students industrial work experience scheme (SIWES) on academic achievement. JEHP 2011;1:80-6.

12. Chiang $Y$, Lee $C$, Lin $Y$, Yen $Y$. Impact of a continuing education program on pharmacists' knowledge and attitudes toward asthma patient care. Med Princ Pract 2010;19:305-11.

13. Basheti IA, Qunaibi EA, Hamadi SA, Reddel HK. Inhaler technique training and healthcare professionals. Effective longterm solution for a current problem. Respiratory Care 2014:59:1716-25

14. Desolu O0, Busari O, Onyedum CC, Salawu FK, Obateru OA, Nwogu KC, et al. Evaluation of current knowledge, awareness and practice of spirometry among hospital-based Nigerian doctors. BMC J 2009;9:6-10.

15. Akram W, Hussein MSE, Ahmad S, Mamat MN, Ismail NE. Validation of the knowledge, attitude and perceived practice of asthma instrument among community pharmacists using Rasch analysis. Saudi Pharm J 2015;23:499-503.

16. Kritikos VS, Reddel HK, Bosnic-Anticevich SZ. Pharmacists' perceptions of their role in asthma management and barriers to the provision of asthma services. Int J Pharm Pract 2010;18:209-16.

17. Odili VU, Ajayi FT. Assessing the knowledge of asthma among community pharmacists in Edo State. Int J Health Sci Res 2009;2:315-21. 